\title{
On the Reconstruction of Characteristic Sources in Helmholtz equations using the Method of Fundamental Solutions
}

\author{
Roberto Mamud ${ }^{1}$ \\ Universidade Federal do Rio de Janeiro, Campus Macaé, Macaé, RJ, Brasil \\ Nilson C. Roberty ${ }^{2}$ \\ Programa de Engenharia Nuclear, COPPE, UFRJ, Rio de Janeiro, RJ, Brasil \\ Carlos J. S. Alves ${ }^{3}$ \\ CEMAT, Instituto Superior Técnico, UTL, Lisboa, Portugal \\ Nuno F. M. Martins ${ }^{4}$ \\ Faculdade de Ciências e Tecnologia, Universidade Nova de Lisboa, Caparica, Portugal
}

\begin{abstract}
In [2] we considered the inverse problem that consists in the determination of the support of characteristic sources in physical phenomena described by the modified and classical Helmholtz equations, from boundary measurements. There we identified the location of the barycenter of a star shaped support establishing a simple formula, and this allows to consider a minimization algorithm to recover the original shape, based on simulations by the method of fundamental solutions. Further numerical experiments that validate the barycenter results and the minimization algorithm are presented.
\end{abstract}

Keywords. inverse source problem; Helmholtz equations; characteristic source; method of fundamental solution

\section{Introduction}

Let $\Omega \subset \mathbb{R}^{N}$ be an open, connected and bounded set with $C^{1}$ boundary, $\partial \Omega$. Given the source term $f \in L^{2}(\Omega)$ and the Dirichlet data $g \in H^{1 / 2}(\partial \Omega)$, consider the following problem

$$
\begin{cases}(-\Delta+\lambda) u=f, & \text { in } \Omega, \\ u=g, & \text { on } \partial \Omega,\end{cases}
$$

where we are considering the cases $\lambda=0$ (Laplace equation), $\lambda=\kappa^{2}>0$ (modified Helmholtz equation), and the usual Helmholtz equation $\lambda=-\kappa^{2}<0$, with $\kappa$ denoting the wave number.

The problem (1) has unique solution, unless for some $\lambda<0$ that are eigenvalues of the Dirichlet-Laplace operator for $\Omega$, [4], and by Trace's Theorem, we can define the Direct

\footnotetext{
${ }^{1}$ rmamud@macae.ufrj.br

2nilson@con.ufrj.br

${ }^{3}$ carlos.alves@math.ist.utl.pt

${ }^{4}$ nfm@fct.unl.pt
} 
Problem as the problem of finding $\frac{\partial u}{\partial \nu} \in H^{-1 / 2}(\partial \Omega)$, with $u \in H^{1}(\Omega)$, from the source term $f$ and Dirichlet data $g$, where $\nu$ is the normal outward unity vector. On the other hand, the Inverse Source Problem for this operator is posed as: Given the Cauchy data $\left\{g, g_{\nu}\right\} \in H^{1 / 2}(\partial \Omega) \times H^{-1 / 2}(\partial \Omega)$, find the source term $f$ and a function $u \in H^{1}(\Omega)$, such that

$$
\begin{cases}(-\Delta+\lambda) u=f, & \text { in } \Omega, \\ u=g, & \text { on } \partial \Omega, \\ \frac{\partial u}{\partial \nu}=g_{\nu}, & \text { on } \partial \Omega,\end{cases}
$$

where $\lambda \in \mathbb{R}$. We can study this problem defining some operators. One of these is the Reciprocity Functional associated to (2) that is defined as

$$
\mathcal{R}[f](v):=\int_{\partial \Omega} u \frac{\partial v}{\partial \nu}-v \frac{\partial u}{\partial \nu} d \sigma=\int_{\partial \Omega} g \frac{\partial v}{\partial \nu}-v g_{\nu} d \sigma
$$

for all test functions $v \in \mathcal{H}_{\lambda}(\Omega):=\left\{v \in H^{1}(\Omega) ;(-\Delta+\lambda) v=0\right\}$. Using Green's Identity, we get

$$
\int_{\partial \Omega} g \frac{\partial v}{\partial \nu}-v g_{\nu} d \sigma=\int_{\Omega} v f d x
$$

for all $v \in \mathcal{H}_{\lambda}(\Omega)$. In [3], it is proved that $\mathcal{H}_{\lambda}(\Omega)$ is homeomorphic to $H^{1 / 2}(\partial \Omega)$. In [6], it is studied the identification of star shaped sources for Poisson equation $(\lambda=0)$.

In next theorem we establish an equivalence between the source reconstruction from the Cauchy data and from the reciprocity functional, where the proof can be found in [2].

Theorem 1.1. The Cauchy data uniquely determines the source $f$ if, and only if, $f$ is uniquely determined by $\mathcal{R}[f](v)$, for all $v \in \mathcal{H}_{\lambda}(\Omega)$.

\subsection{The Equivalence between Inverse Characteristic Source Problem and Inverse Jump Problem}

Consider the problem (1) with source $f=h \chi_{\omega}$, where $h \neq 0$ is constant and $\omega \subset \Omega$ and $\Omega \backslash \bar{\omega}$ connected sets. Note that we can rewrite this problem as the transmission problem

$$
\begin{cases}(-\Delta+\lambda) u^{-}=h, & \text { in } \omega, \\ (-\Delta+\lambda) u^{+}=0, & \text { in } \Omega \backslash \bar{\omega}, \\ {[u]=0,} & \text { on } \partial \omega, \\ {\left[\frac{\partial u}{\partial \nu}\right]=0,} & \text { on } \partial \omega, \\ u^{+}=g, & \text { on } \partial \Omega,\end{cases}
$$

where $[u]=u^{-}-u^{+}$denotes the difference between the inner and outer parts of the solution, respectively. Let $\phi$ be a particular solution of the equation $(-\Delta+\lambda) \phi=h$. Remark that if $\lambda \neq 0$, we can take $\phi=h / \lambda$ and if $\lambda=0$, we can take $\phi(x)=h\|x\|^{2} / 4$. Thus, considering

$$
\vartheta= \begin{cases}u^{+}, & \text {in } \Omega \backslash \bar{\omega} \\ u^{-}-\phi, & \text { in } \omega\end{cases}
$$


we have that, up to $\phi$, the problem (5) is equivalent to the inverse jump problem

$$
\begin{cases}(-\Delta+\lambda) \vartheta=0, & \text { in } \Omega \backslash \partial \omega, \\ {[\vartheta]=-\phi,} & \text { on } \partial \omega, \\ {\left[\frac{\partial \vartheta}{\partial \nu}\right]=-\frac{\partial \phi}{\partial \nu},} & \text { on } \partial \omega, \\ \vartheta=g, & \text { on } \partial \Omega .\end{cases}
$$

Thus, denoting the reciprocity functional of this problem as $\mathcal{R}[\partial \omega](\cdot)$, we establish the following result, where the proof can be found in [2].

Theorem 1.2. If $\Omega \backslash \bar{\omega}$ is connected, then $\mathcal{R}\left[\chi_{\omega}\right]=\mathcal{R}[\partial \omega]$. Therefore, the inverse source problem of characteristic source (1) is equivalent to the inverse jump problem (7).

\section{Centroid Determination in Characteristic Sources}

Consider $\kappa=\sqrt{\lambda} \neq 0$, where $\kappa \in \mathbb{R}$, if $\lambda>0$, and $\kappa \in \mathbb{C} \backslash \mathbb{R}$, if $\lambda<0$. Consider also the source term $f(x)=\chi_{\omega}(x)$, where $\chi_{\omega}$ is the characteristic function of a open, connected, bounded subset, $\omega \subset \Omega$, with regular boundary, $\partial \omega$, for the inverse source problem (2), and $\mathbb{S}^{N-1}$ is the boundary of the open unity ball in $\mathbb{R}^{N}$.

So, taking the test function $v_{\varphi}(x)=e^{\kappa \varphi \cdot(x-p)}$, where $\varphi \in \mathbb{S}^{N-1}$ and $p \in \mathbb{R}^{N}$ are arbitrary, the reciprocity functional for problem (2) is given by definition

$$
\mathcal{R}\left[\chi_{\omega}\right]\left(e^{\kappa \varphi \cdot(x-p)}\right)=\int_{\partial \Omega} g \kappa(\varphi \cdot \nu) e^{\kappa \varphi \cdot(x-p)}-g_{\nu} e^{\kappa \varphi \cdot(x-p)} d \sigma .
$$

Remark 2.1. Denoting $\mathcal{R}\left[\chi_{\omega}\right](\varphi):=\mathcal{R}\left[\chi_{\omega}\right]\left(e^{\kappa \varphi \cdot x}\right)$, we have

$$
\mathcal{R}\left[\chi_{\omega}\right]\left(e^{\kappa \varphi \cdot(x-p)}\right)=e^{-\kappa \varphi \cdot p} \mathcal{R}\left[\chi_{\omega}\right](\varphi)
$$

The proof of the following results can be found in [2].

Theorem 2.1. Let $\omega \subset \Omega$ be a star shaped subset and let $f(x)=\chi_{\omega}(x)$ be the source term for the inverse problem (2). Then the centroid, $p$, of $\omega$ can be determinate by

$$
\varphi \cdot p=\frac{1}{2 \kappa} \ln \left(\frac{\mathcal{R}\left[\chi_{\omega}\right](\varphi)}{\mathcal{R}\left[\chi_{\omega}\right](-\varphi)}\right)
$$

where $\varphi \in \mathbb{S}^{N-1}$.

Corollary 2.1. Let $f_{1}=\chi_{\omega_{1}}$ and $f_{2}=\chi_{\omega_{2}}$ two characteristic sources for the inverse problem (2), with $\omega_{1}, \omega_{2} \subset \Omega$ star shaped subsets. If these sources generate the same Cauchy data on the boundary, then these sources have the same centroid given by (8). 


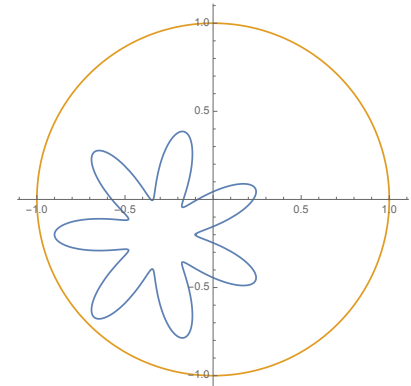

Figure 1: Problem Domain - 7-Star inside Circle

\subsection{Numerical Experiments for Centroid Determination}

In this experiment was considered the domain $\Omega$ as the interior of the circle parametrized by $R(t)=(\cos (t), \sin (t)), t \in[0,2 \pi]$, and the support of the source as the star shaped set $\omega$, with centroid $\left(x_{c}, y_{c}\right)=(-0.3,-0.2)$, which boundary, $\partial \omega$, is parametrized by $r(t)=\left(x_{c}, y_{c}\right)+(0.4-0.2 \cos (7 t))(\cos (t), \sin (t)), t \in[0,2 \pi]$, as shown in figure 1 , with null Dirichlet data. It was considered 500 collocation points and 250 source points in the MFS. The centroid was determined supposing relative noise over the Neumann data, where the position error was calculated by the Euclidean distance between the original point and the reconstructed by the formula. The result obtained is shown in table 1 .

Table 1: Centroid Error - 7-Star Shaped Case.

\begin{tabular}{|l|c|c|}
\hline & Centroid Calculated & Position Error \\
\hline Noise 0\% & $(-0.300041,-0.200032)$ & $5.20267 \times 10^{-5}$ \\
\hline Noise 1\% & $(-0.300193,-0.20007)$ & $2.0509 \times 10^{-4}$ \\
\hline Noise 5\% & $(-0.299697,-0.200433)$ & $5.28746 \times 10^{-4}$ \\
\hline Noise 10\% & $(-0.298261,-0.199676)$ & $1.76905 \times 10^{-3}$ \\
\hline
\end{tabular}

\section{Boundary Reconstruction Using MFS}

The method of fundamental solutions (MFS) is a technique used to find numerical solution of certain boundary value problems (e.g. [5]). In this work, we follow the ideas of [1] to formulate the MFS solution expansion.

Consider the direct problem related to Laplace-Helmholtz equation

$$
\begin{cases}(-\Delta+\lambda) u=f, & \text { in } \Omega, \\ u=g, & \text { on } \partial \Omega .\end{cases}
$$

where the source term $f=h \chi_{\omega}$, with $h \neq 0$ constant and $\omega \subset \Omega$ an open, connected, bounded subset with boundary $C^{1}, \partial \omega$. 
Observe that, by subsection 1.1, the problem (9) can be rewritten as the transmission problem for $\vartheta,(7)$, and $u^{+}$and $u^{-}$will be determined by the Method of Fundamental Solution (MFS). For this, consider the following expansions centred at the corresponding source points

$$
u^{+}(x)=\sum_{j=1}^{q} \beta_{j}^{I} \Phi_{\lambda}\left(\sqrt{\lambda}\left|x-a_{j}\right|\right)+\sum_{l=1}^{r} \beta_{l}^{E} \Phi_{\lambda}\left(\sqrt{\lambda}\left|x-b_{l}\right|\right), \quad u^{-}(x)=\sum_{i=1}^{p} \alpha_{i} \Phi_{\lambda}\left(\sqrt{\lambda}\left|x-c_{i}\right|\right),
$$

with $a_{j} \in \Gamma^{I}, b_{l} \in \Gamma^{E}$ and $c_{i} \in \gamma$, where $\Phi_{\lambda}$ is a fundamental solution of Laplace-Helmholtz equation, that is, $(-\Delta+\lambda) \Phi_{\lambda}=\delta$, where $\delta$ is the Dirac distribution. Beside this, the fictitious boundary $\gamma, \Gamma^{I}$ and $\Gamma^{E}$ are smooth and chosen such that: The curve $\gamma$ be outer to $\omega$, with $\gamma \subset(\Omega \backslash \bar{\omega})$; The curve $\Gamma^{I}$ be outer to $\Omega \backslash \bar{\omega}$, with $\Gamma^{I} \subset \omega$; The curve $\Gamma^{E}$ be outer to $\Omega \backslash \bar{\omega}$, with $\Gamma^{E} \subset \Omega^{c}$. So, for be a solution of (7) is necessary that $\vartheta$ also satisfies the boundary conditions. In this way, taking collocation points $x_{m^{*}} \in \partial \omega$, with $m^{*}=1,2, \ldots, m$, and $x_{n^{*}} \in \partial \Omega$, with $n^{*}=1,2, \ldots, n$, we can write the above problem as a linear system, $M . \alpha=b$, where $\alpha$ is the vector formed by the coefficients, to be determined, of MFS expansion, $b$ is the vector formed by the boundary conditions and $M$, $(2 m+n) \times(p+q+r)$, is formed by the linear system coefficients. In general, it is considered $2 m+n \geq 2(p+q+r)$ in characterization of matrix $M$. Therefore, solving the linear system $M . \alpha=b$, we find a solution, $\vartheta$, of $(7)$, in which we generate the artificial data $\frac{\partial \vartheta}{\partial \nu}$, on $\partial \Omega$, to be used in the inverse problem for determine the support $\omega$ by Levenberg-Marquardt algorithm.

\subsection{Numerical Experiment for Boundary Reconstruction}

Consider the source term $f(x)=\chi_{\omega}(x)$, where $\omega \subset \Omega$ is an open, connected and bounded subset of $\Omega \subset \mathbb{R}^{2}$. Consider the boundary, $\partial \omega$, parametrized by function $R(t)$. Suppose this function can be written as

$$
R(t)=\left(x_{c}, y_{c}\right)+\left(r_{M}+\sum_{n=1}^{\infty} \alpha_{n} \cos \left(\frac{n t}{2}\right)+\alpha_{n+1} \sin \left(\frac{n t}{2}\right)\right)(\cos (t), \sin (t)),
$$

with $t \in[0,2 \pi]$, where $\left(x_{c}, y_{c}\right)$ is the centroid of $\omega$, given by the new reconstruction formula (8), and $r_{M}$ is the initial guess in the method, called mean radius of $\omega$, and it is related to the intensity of a single point source. Consider the reciprocity functional $\mathcal{R}\left[\chi_{\tilde{\omega}}\right]$, where $\tilde{\omega} \subset \Omega$ is an approximation of $\omega$, with $\partial \tilde{\omega}$ parametrized by (10). So, taking the test function $v_{\theta_{i}}(x)=e^{\kappa\left(\cos \theta_{i}, \sin \theta_{i}\right) \cdot x}$, with $M$ different directions where $\theta_{i}=i \frac{2 \pi}{M}, i=1, \ldots, M$, and $N P$ as number of parameters in Fourier expansion, the main goal is minimize, over the set of parameters $\alpha_{n}, n \in\{1, \ldots, N P\}$, the functional

$$
J\left(v_{\theta_{i}}\right)=\sum_{i=0}^{M}\left(\mathcal{R}\left[\chi_{\omega}\right]\left(v_{\theta_{i}}\right)-\mathcal{R}\left[\chi_{\tilde{\omega}}\right]\left(v_{\theta_{i}}\right)\right)^{2} .
$$

Experiment $-\lambda=1$ : In this experiment, it was considered an original support, $\omega$, whose boundary, $\partial \omega$, is parametrized by $r(t)=\left(x_{c}, y_{c}\right)+(0.3-0.15 \cos (5 t))(\cos (t), \sin (t))$, 
$t \in[0,2 \pi]$, where $\left(x_{c}, y_{c}\right)=(-0.3,0.1)$, and the domain as the interior of circle parametrized by $R(t)=(\cos (t), \sin (t)), t \in[0,2 \pi]$, as shown in figure 2 , where the Dirichlet data was considered null. Beside this, it was considered the number of parameters in Fourier expansion as 11 , that is, $N P=11$, and $M=24$. In original problem was considered 240 collocation points and 120 source points and in approximate problem, 200 collocation points and 100 source points. The initial parameter vector in reconstruction was $\left(r_{M}, 0,0,0,0,0,0\right)$, with mean radius $r_{M}=0.320988$. After 10 iterations, of approximately $52 \mathrm{~s}$ each, it was obtained the reconstruction with and without noise shown in figure 2 , where the figure in red is the original and in green is the reconstructed.

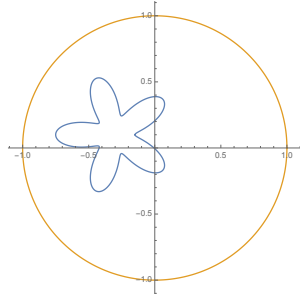

(a) Domain/ Support

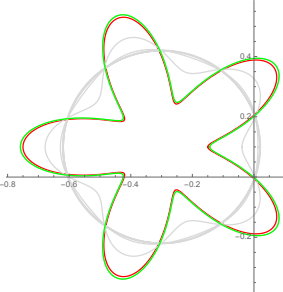

(b) No noise

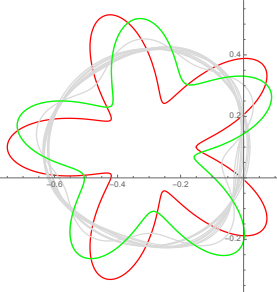

(c) $5 \%$ relative noise

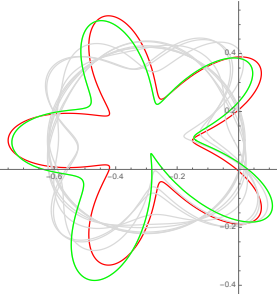

(d) $10 \%$ relative noise

Figure 2: Case $\lambda>0$. Experiment with domain of radius 1

Experiment $-\lambda=-1$ : In this experiment, it was considered an original support, $\omega$, whose boundary, $\partial \omega$, is parametrized by $r(t)=\left(x_{c}, y_{c}\right)+(0.3-0.15 \cos (3 t))(\cos (t), \sin (t))$, $t \in[0,2 \pi]$, where $\left(x_{c}, y_{c}\right)=(-0.3,0.1)$, and the domain, $\Omega$, as the interior of the circle parametrized by $R(t)=(\cos (t), \sin (t)), t \in[0,2 \pi]$, as shown in figure 3 , with null Dirichlet data. It was considered the number of parameters in Fourier expansion as 7 , that is, $N P=7$, and $M=36$. The mean radius, was $r_{M}=0.315245-8.11401 \times 10^{-8} i$. In original problem was considered 200 collocation points and 100 source points and in approximate problem, 160 collocation points and 80 source points. Beside this, the fundamental solution was taken different because $\lambda<0$, and the test function was taken $K_{0}\left(\sqrt{-\lambda}\left|x_{m}-a\right|\right)$, where $x_{m}$ is a collocation point and $a$ is a source point. After 5 iterations, of approximately $65 \mathrm{~s}$ each, it was obtained the reconstruction with and without noise shown in figure 3, where the figure in red is the original and the figure in green is the reconstructed. Although the time, in each iteration, the reconstruction is greater than the previous experiment, where the convergence is faster, because from the third iteration there are not visual differences between each reconstruction.

Remark 3.1. To avoid problems known as "inverse crimes", the MFS used for the simulation of the direct problem was different from the one used for the inverse iterations.

\section{Conclusions}

In this work, based on [2], we studied the inverse characteristic source problem for Helmholtz equations. Considering $\omega$ the support of characteristic source as an open, connected, bounded subset of the domain, we present new numerical experiments related 


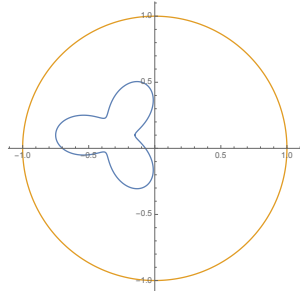

(a) Domain/ Support

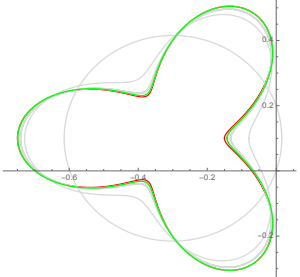

(b) No noise

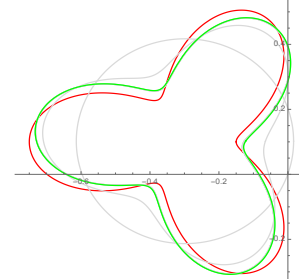

(c) $5 \%$ relative noise

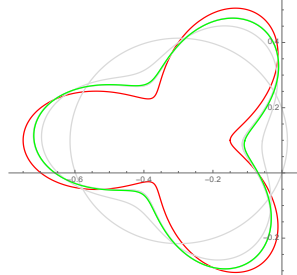

(d) $10 \%$ relative noise

Figure 3: Case $\lambda<0$. Experiment with domain of radius 1

to centroid and boundary reconstruction of $\omega$. These new experiments complement the validation of centroid formula proposed in [2] and the respective numerical method used.

\section{Acknowledgements}

This work was partially financed by Brazilian agencies CNPq, process number 141829/2012-5 and 305080/2013-0, and CAPES, process number 99999.007941/2014-5.

\section{References}

[1] C. J. S. Alves, C. S. Chen. A new method of fundamental solutions applied to nonhomogeneous elliptic problems. Advances in Computational Mathematics, 23: 125-?142, 2005.

[2] C. J. S. Alves, R. Mamud, N. F. M. Martins, N. C. Roberty. Reconstruction of Characteristic Sources in Helmholtz equations using the Method of Fundamental Solutions. Submitted to Inverse Problems in Science and Engineering, 2016.

[3] C. J. S. Alves, N. F. M. Martins, N. C. Roberty. Full identification of acoustic sources with multiple frequencies and boundary measurements. Inverse Problems and Imaging, 3: 275-?294, 2009.

[4] L. Evans. Partial Differential Equations. Graduate Studies in Mathematics, 19. Berkeley: University of California, 2010.

[5] G. Fairweather, A. Karageorghis. The method of fundamental solutions for elliptic boundary value problems. Advances in Computational Mathematics, 9: 69-95, 1998.

[6] N. C. Roberty, C. J. S. Alves. On the identification of star shape sources from boundary using a reciprocity functional. Inverse Problems in Science and Engineering, 17: 187-202, 2009. 